\title{
What happens when a climber falls? Young climbers mathematise a climbing situation
}

\author{
Anne Birgitte Fyhn ${ }^{1}$ \\ ${ }^{1}$ Department of education, UiT - The Arctic University of Norway, Tromsø, Norway \\ For correspondence: anne.fyhn@uit.no
}

\begin{abstract}
Students in Norway and other countries experience vectors as a difficult topic. Four young skilled climbers, who all did well in mathematics at school, participated in the Vector Study (VS). They participated for free and each lesson lasted until the students decided it was over. The idea was to investigate how climbing may function as a basis for students' development of a vector concept. The teaching goal was the parallelogram law of vector addition. The students investigated what happens when a climber falls. They discussed the situation, and they tested it out in practice. They also performed a supporting activity, a rope-pulling situation, which provides insight into what happens in the climbing situation. The students' development is analysed by identifying a) Bishop's six basic activities, b) the role of angles, and c) manipulation of mental objects. The analysis reveals that relations between a vector's magnitude and direction was central in the students' investigations. It is important that students develop two aspects of vectors before the parallelogram law of addition is introduced. These are a) relations between angles and vectors, and b) the zero vector.
\end{abstract}

Keywords: climbing, vector, mathematisation

\section{Introduction}

In Norway, vectors are part of the mathematics syllabus for the second year of upper secondary school, and students experience it as being a difficult theme (Fyhn, 2011). In England, vectors are introduced some years earlier, and according to Poynter and Tall (2005) many students there experience vectors as difficult. Rosenbloom (1969) highlighted the role of the students' geometric intuitions in the introduction to vectors, as a response to many authors who back then gave overly abstract presentations of vectors with heavily algebraic treatments. The Vector Study (VS) intends to build on students' geometrical intuitions. Four young climbers were introduced to vectors in their spare time over the course of three afternoon lessons. They had not yet been introduced to vectors in school. The aim of the VS is to provide insight into how students' climbing experiences may function as a basis for introducing vectors. Persons who climb do it for fun and not for any external reward; they climb because the activity is exciting and interesting. This is a contrast to the traditional mathematics lessons at school, where many students participate for other reasons than intrinsic motivation.

According to Bishop (1988), mathematics was considered to be culture free until the 1980s. He argued that just as each cultural group have developed their language and religion, they have also developed their mathematics. Klausen (1992) defines 'culture' as the ideas, values, rules, norms, codes and symbols that a person adopts from the previous generation and that one tries to transmit more or less unchanged to the next generation. According to Eriksen (1997), culture is the common thought patterns, habits and experiences that make it possible for human beings to understand each other; it is what makes communication possible. We consider culture to be something personal as well as 
something social. Thus, climbing culture consists of ideas, values, norms, rules, codes and symbols that one generation of climbers adopts from the previous generation; it is what makes climbing communication possible. The VS focuses on vectors, which have or might have developed from climbing culture.

Gay (2016) introduced culturally responsive teaching, which involves «using the cultural knowledge, prior experiences, frames of reference, and performance styles of ethnically diverse students to make learning encounters more relevant to and effective for them» (p. 50-51). The VS is culturally responsive teaching, because the study uses the participating climbers' cultural knowledge, prior experiences and frames of reference in order to contribute to making the learning encounters relevant for them.

According to Dewey (1998/1933), mastery of the body is an intellectual problem. He claims that the joy the child shows in learning how to use its limbs, to translate what she/he sees into what she/he handles, is sufficient evidence for the claim that the development of physical control is an intellectual achievement. Climbing is mastery of body, and climbing is thus an intellectual activity. Fyhn (2006; 2008) investigated young climbers' reflections about angles. The VS takes this approach further and focuses on older youths' investigations of a climbing situation where vectors are an expected learning outcome.

Freudenthal (1973) introduced the term 'guided re-invention,' where the idea is that the teacher guides the students through a re-invention of mathematical concepts. The teaching method of reinvention is built on interpreting and analysing mathematics as an activity, and a mathematical activity is explained as organising fields of experience. The students should also learn to organise unmathematical matters into a structure that is accessible to mathematical refinements. This is referred to as 'mathematising'. The field of experience to be organised is students' climbing culture, and the expected learning outcome is an increased understanding of what happens when a climber falls. Vectors might be a tool for understanding falling situations. Bishop (1988) also relates mathematics to activity. He claims that mathematics is a cultural product that has developed from six different activities. Freudenthal (1983) underlines the importance of starting with the phenomena that beg to be organised, and «from that starting point teaching the learner to manipulate these means of organizing» (p. 32). Thus, the VS does not start with vectors and look for a way to concretise this abstraction. The idea is rather to take the students' climbing experiences as the point of departure and find a way to guide them towards grasping a vector concept that may be a useful tool in their climbing culture. The study does not aim to create a teaching approach suitable for everyone; the goal is to create an approach to vectors that can make sense to climbers who are familiar with what goes on in their real world.

The research question is, what potential does the mathematisation of climbing have for climbers' development of a vector concept, and what are the limitations? The idea was to guide the students through mathematisation of a well-known situation from spare time climbing. The lessons started with performing the activities. Afterwards the students sat down to draw, describe and explain what they had done. The study is based on the situation showed in Figure 1. Here Stian, the climber, wears a harness tied to one end of the rope. The rope goes down to Lars, the 'belayer', who has attached the rope to a breaking device in his harness. As the climber proceeds upwards, the belayer is paying out rope through the breaking device. The climber regularly 'clips' the rope onto carabiner hooks, 'runners', which are attached to the wall. When the climber falls, he passes the last runner he clipped and ends up hanging from it. The fall stops when the rope is tightened. The belayer experiences a tug from the rope and he might be lifted slightly off the ground. The study focuses on mathematisation of two situations: a) a falling situation similar to the climbing example in Figure 1, where the goal is to 
explain what happens to the belayer when the climber falls and why; and b) a rope-pulling situation, which was chosen in order to support the students' explorations of the falling situation.
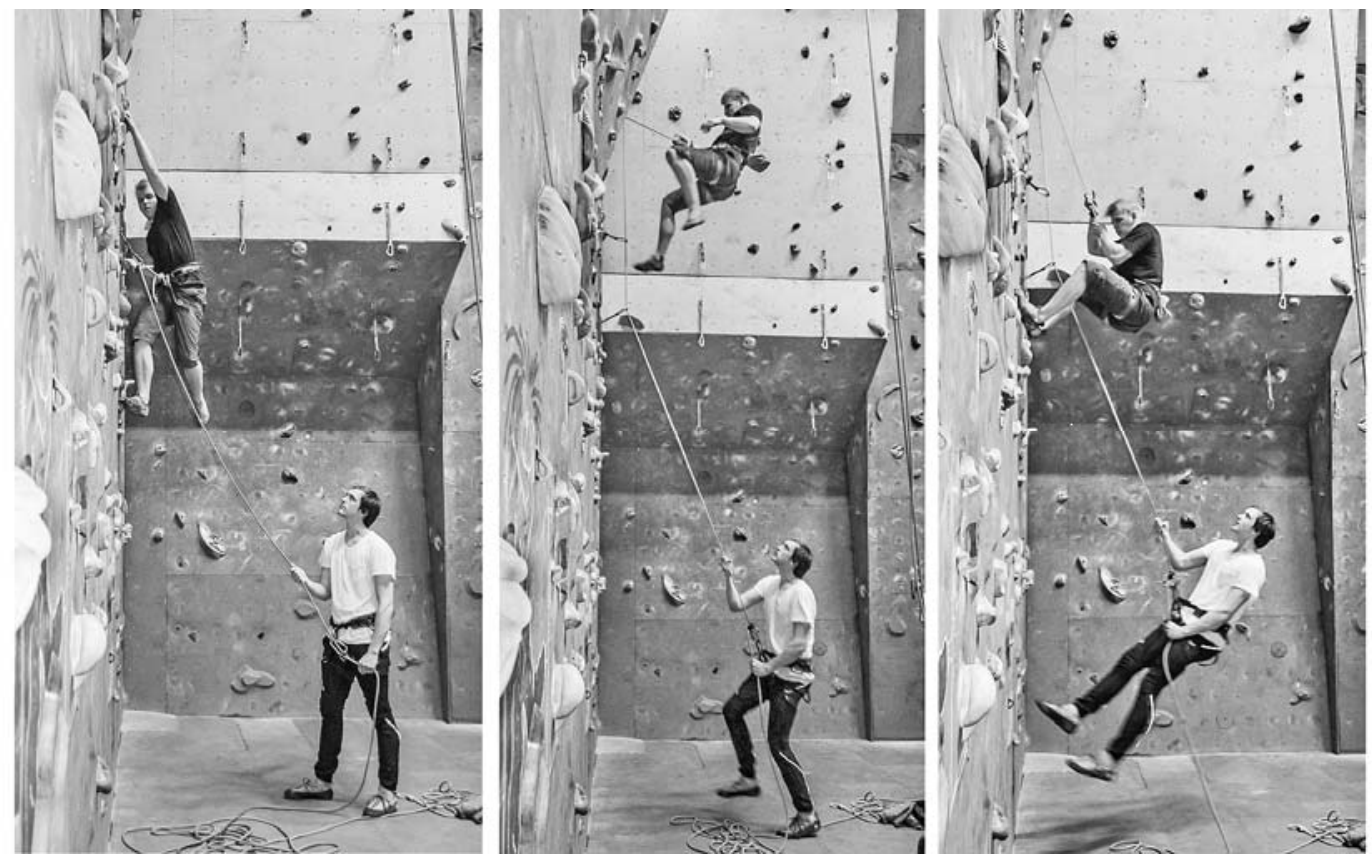

Figure 1. Lead climbing and falling on a climbing wall

Hayfa (2006) investigates the impact of language and notation on the conceptualisation of vectors in Lebanese schools. She refers to regular geometry practice. The VS focuses neither on notation nor regular geometry practice but on the students' context-bound, intuitive ideas and reasoning. There is much evidence of learning difficulties connected to vectors and linear algebra. Research on French students who had completed their first university course on linear algebra reveals numerous difficulties at different levels (Harel and Trgalová, 1996). Among other things, the students had difficulties understanding the concept vector space. In the US and Canada the $\mathbb{R}^{\mathrm{n}}$ model and the matrix theory are more predominant in the early linear algebra courses. There are serious difficulties associated with the teaching of linear algebra in North America. Maracci (2006) researched underand postgraduate students' errors and difficulties in solving problems related to vector space theory. Different students came up with similar errors. The difficulties concerned linear combination, linear independence, basis and spanning set, which are basic notions of vector space theory. The findings support the idea that the basic introduction of vectors needs to have a focus on concept development.

Freudenthal (1973) introduced the term 'mathematisation'. To mathematise an unmathematical or insufficiently mathematical matter means learning to organise it into a structure that is accessible to mathematical refinements. Freudenthal (1991) shares Bishop's activity perspective of mathematics; mathematics is not a ready-made system, but «an activity of discovering and organising in an interplay of content and form» (p. 15). Freudenthal (1973) underlines the importance of students learning to mathematise, particularly at the lowest level, where mathematisation is applied to unmathematical matters. Freudenthal's conception of a level structure stems from his cooperation with the van Hieles. According to van Hiele (1986), we can distinguish between five levels of mathematical thinking. The move from one level to the next is not a natural process; it takes place under the influence of a teaching-learning programme. The move to a higher level involves a change in language. The levels are: level 1, the visual level; level 2, the descriptive level; level 3: the logical relations level; level 4: the formal logic level; and level 5: the nature of logical laws. At the first level, the student can say «this is a rhombus,» and another student can follow up and claim that «this is a rhombus, too!» However, it makes no sense to ask them why. «There is no why, one just sees it», (p. 
83). The discussion and understanding of such questions require the language belonging to the descriptive level. True learning at one level is not possible as long as the learning process at the lower level has not been sufficiently completed. This paper focuses on students who are not familiar with vectors. They start out below the first level, which means that they are unable to make claims like, «that is a vector!» Statements like «this is a vector because ...» belong to level 2, the descriptive level, so one challenge in teaching vectors is to start out with a language that can lead to level 1 , and avoid rushing to level 2 before level 1 has been achieved. The VS departs from the students' climbing language and their climbing thoughts, which are intertwined with the climbing context. During the VS, students learn to mathematise an unmathematical subject matter, falling situations, which is central to their climbing culture.

\section{Theoretical framework}

The framework for the VS is constituted by identification of Bishop's (1988) six basic activities and the role of angles in vector concept development, which Poynter and Tall (2005) and Georgios, Panayotis and Athanasios (2005) point out as being important. Mathematical concepts, structures and ideas serve to organise phenomena in the concrete world as well as in mathematics (Freudenthal, 1983). The constitution of mental objects precedes concept attainment. When children learn what numbers, directions and addition are, they grasp these items as mental objects and carry them out through mental activities. Manipulation of mental objects is an activity that precedes concept attainment.

Six basic activities.

According to Bishop (1988), there are six basic activities, which are carried out in every cultural group, that are both necessary and jointly sufficient for the development of mathematical knowledge. The activities are: Counting, Locating, Measuring, Designing, Playing and Explaining. Explaining and locating play the main roles in the VS, but the other activities are present, too. Explaining refers to finding ways to account for the existence of phenomena. Explaining does not refer to the explanation of vectors, because vectors are the learning outcome and not something that is part of climbing culture. The phenomena that need to be explained are how a climber falls and how a belayer is moved when a climber falls. Locating is «[e]xploring one's spatial environment and conceptualizing and symbolizing that environment, with models, diagrams, drawings, words or other means» (p. 182). This is largely what the VS is concerned with. The aim of the VS is that such exploration will result in a more or less formalised vector concept. Counting refers to a systematic way of comparing and ordering discrete phenomena. The discrete phenomena to be ordered in indoor climbing are individuals, hands, feet or ropes. Counting is expected to play a minor role in the VS. Measuring concerns quantifying qualities for the purposes of comparison and ordering. In indoor climbing, this means comparisons of forces» magnitudes and directions. Designing means creating a shape or design for an object or for any part of one's spatial environment. This is what happens when climbers discuss what goes on in a climbing situation and make drawings, gestures or models to illustrate their ideas of what is going on. Playing is important in climbing. A typical rule of the game is to climb up an indoor wall using holds of only one colour. The participants can make their own rules, such as banning footholds or introducing a time limit for completing a route.

The role of angles

Poynter and Tall (2005) investigated teachers' ideas about students' difficulties in developing a vector concept. They claim that «[t]he goal is to create conceptual knowledge with a relational understanding of the concepts ...» (p. 133). In addition, the parallelogram law, the triangle law, and the addition of vector components have to appear as different aspects of the same concept. The VS builds on recommendations from Poynter and Tall (2005) because it aims to provide new insight into how students learn, and focuses on relations between the concepts vector and angle. The importance of 
these relations is also supported by Georgios, Panayotis and Athanasios (2005), who claim that the role of angles in cognitive procedures concerning vectors has been underestimated.

\section{Method}

The VS is a low-cost project where the participating teacher is the researcher. The lessons took place in a climbing hall and in adjacent rooms, all located in walking distance from the researcher's office. Negative aspects of such a low-cost project include a limited amount of data and the fact that no other researchers took part in discussing the analyses. There is a risk that the researcher misses some important aspects that would have surfaced during a discussion. A positive aspect of a singleresearcher-project is that it is easier for one person to exhibit consistent behaviour throughout the lessons; nor is there any lack of information between the researchers. The VS context lacks the usual mathematics classroom constraints, such as students wanting to do something else or students who behave in ways they believe will please the teacher. The students participated out of an interest in climbing. They showed up, they participated in rope-pulling activities and in dialogues, and they made drawings. They also laughed a lot. Thus, there are reasons to believe that the students reflected on what they did.

The design is based on the idea of finding a 'classic situation' related to the students' leisure time activity. The situation has to open for an investigative approach. The goal of the investigations is to describe and analyse what happens in order to search for a better understanding of what goes on. In order to control for a culturally responsive mathematics teaching, as described by Gay (2016), the mathematics teacher must either belong to the culture under investigation or cooperate closely with cultural experts. The basis for the design is investigations of a 'classic situation' within the culture. The design can easily be replicated by researchers who enjoy climbing. This design constitutes the basis for other studies involving a) students in a Sámi middle school who investigated Sámi braidings (Fyhn et al., 2015a), b) a secondary school music program where drummers investigated 'division levels' and string instrument players investigated 'overtones' (Fyhn et al., under review a), c) students in a Sámi primary school who investigated Sámi ornamentations (Fyhn et al, 2015b), and d) grade three students in primary school investigating where to stand in order to score goals in soccer and how to sing 'Father Jacob' and other songs in canon (Fyhn et al., under review b).

\section{A descriptive case study}

The VS is a case study (Yin, 2009) of four young students who took part in three afternoon lessons. Each lesson started at a given time, and lasted as long as the participants found it interesting between two and three hours. The lack of elements that control behaviour means that the current study is a case study and not an experiment. The researcher was involved in what goes on; she was not a silent observer. The intention is to shed light on a complex social phenomenon: what happens when a group of four young climbers are invited to investigate what really goes on in a classic climbing situation. A case study allows the investigation to maintain the holistic and meaningful characteristics of real-life events. The study is descriptive, because it does not aim to explain why things happen; the main purpose is to describe an example of how the complex process of teaching and learning vectors may take place.

The four students volunteered to participate in the VS in their spare time, knowing that they could leave whenever they wanted. Situations may arise where one student experiences something as being embarrassing due to a lack of some piece of knowledge or skill in mathematics. The researcher needs to avoid such situations. Three of the students were recruited among the participants in a local climbing competition: Lars and Stian are last-year students in lower secondary school, while Øystein is a first-year student in upper secondary school. Martine was asked to participate because she is a member of the local climbing club's board, and she is a first-year student in upper secondary school. 
The students attend four different schools. Two of them knew the researcher well from climbing. Martine joined the group starting with Lesson 2; Lars was absent from Lesson 2, while Øystein was absent from Lesson 3.

The students' mathematisation of the falling situation in Figure 1 is the focus of the VS. The study takes as its point of departure a climbing problem that is trivial to climbers: Where to locate yourself while your friend is climbing up a wall and you are tied into the other end of the rope? The explanation for why is non-trivial. The students have to experience standing close to the wall while belaying, as well as standing a bit away from the wall. The basis is the students' know-how, their knowledge of where to locate themselves. One opportunity arising when the falling situation is used as a basis for teaching is that the students will experience the situation again and again as an integrated part of their climbing. Thus, the ground is prepared for students' reflections around why things happen. Traditional textbook-based teaching does not provide this opportunity. According to Dewey (1933/1998), reflection begins when we start inquiring into the reliability or worth of something - in other words, when we try to see what guarantee there is that the existing data really point to the idea that is suggested. Reflection accordingly implies that something is believed in. "One can think reflectively only when one is willing to endure suspense and to undergo the trouble of searching» (p. 16).

In order to illuminate the falling-and-belaying situation from one more perspective, the task in Figure 2 was added. The task is a development of a Norwegian textbook task by Andersen et al. (2007, pp. 47-48), and there is thus a risk that this task is not culturally responsive. If this task turns out to support the students' explanations of the falling situation, the task shows culturally responsiveness.

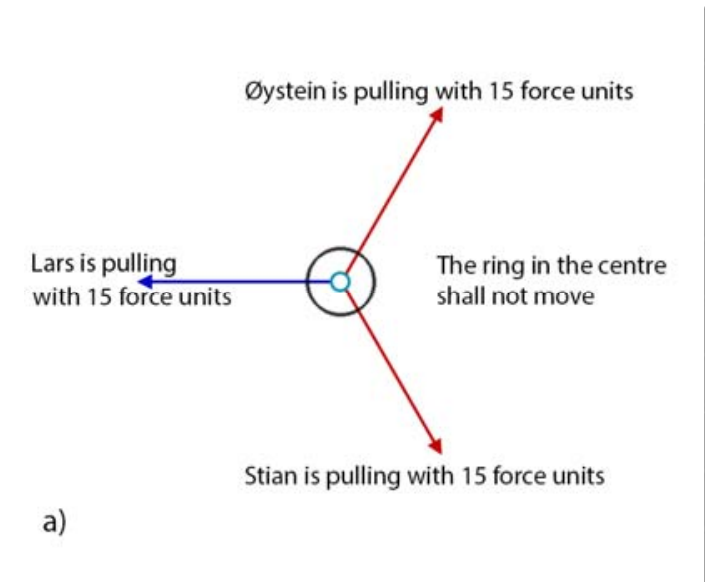

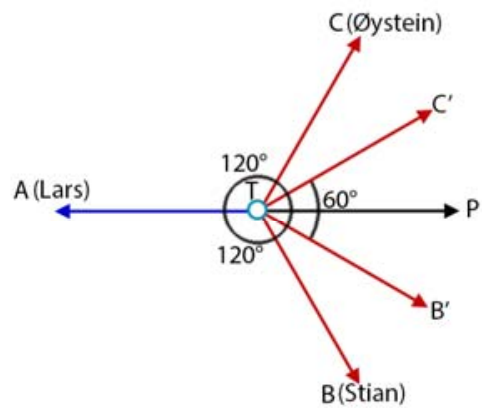

b)

Figure 2. The rope-pulling task

Lars (A), Stian (B) and Øystein (C) have fastened three ropes to a ring. In Figure 2a, the boys pull one rope each, and they pull equally hard on their ropes. The ring is not supposed to move. The question is: what is the angle between two ropes? Figure $2 \mathrm{~b}$ includes two tasks: (1) «Imagine that we substitute Stian's and Øystein's forces by one force that works in an opposite direction to Lars' force: the new force is $\mathrm{P}$ in the figure. The ring is still not supposed to move. Explain how strong this new force will have to be.» (2) «Lars's force is unchanged and the ring still does not move. Stian and Øystein pull with equal force while the angle between their ropes is $60^{\circ}$. What is the magnitude of their forces when they are located in $\mathrm{B}^{\prime}$ and $\mathrm{C}^{\prime}$ ?» The researcher expected this task to give the students insight into the role of angles. Here the climbers wear climbing harnesses and use climbing ropes and carabiners, which are codes and symbols that belong to climbing culture. The task is a closed experiment, where the focus is on how the force from a rope changes when the rope's direction (angle) is changed. The situation is based on a textbook task. It is mathematisation of a situation from 'a semi-reality', while the falling situation involves investigations of the students' real-life 
experiences. Because the task does not belong to climbing reality, there is a risk that this situation will not contribute to young climbers' learning.

Teaching goal, plan and data

Poynter and Tall (2005) introduced vectors as displacement, while the VS introduces vectors as force. As pointed out by Georgios, Panayotis and Athanasios (2005) the vector as a journey leads more naturally to the use of the triangular law, while vectors as force leads to the parallelogram law. The VS teaching goal is accordingly the parallelogram law of vector addition, presented in Figure 3. The sum $A+B$ of the vectors is obtained by placing them head to tail and drawing the vector from the free tail to the free head; The sum $A+B$ is constituted by the parallelogram's diagonal.
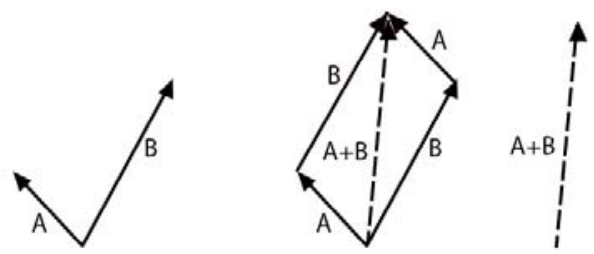

Figure 3. The parallelogram law gives the rule for vector addition of vectors $A$ and $B$.

There was one week between Lessons 1 and 2, and there were two weeks between the last lessons. Lesson 1 started with a short presentation of the participants and the VS. Then the students wrote down what they knew from before about vectors and geometry. The students were going to experience both falling and belaying in a climbing situation like the one described in Figure 1. Any climber knows that in lead climbing the belayer has to stand close to the wall to avoid being tugged up and into the wall if a fall occurs. This is basic climbing knowledge, but explaining why is not basic.
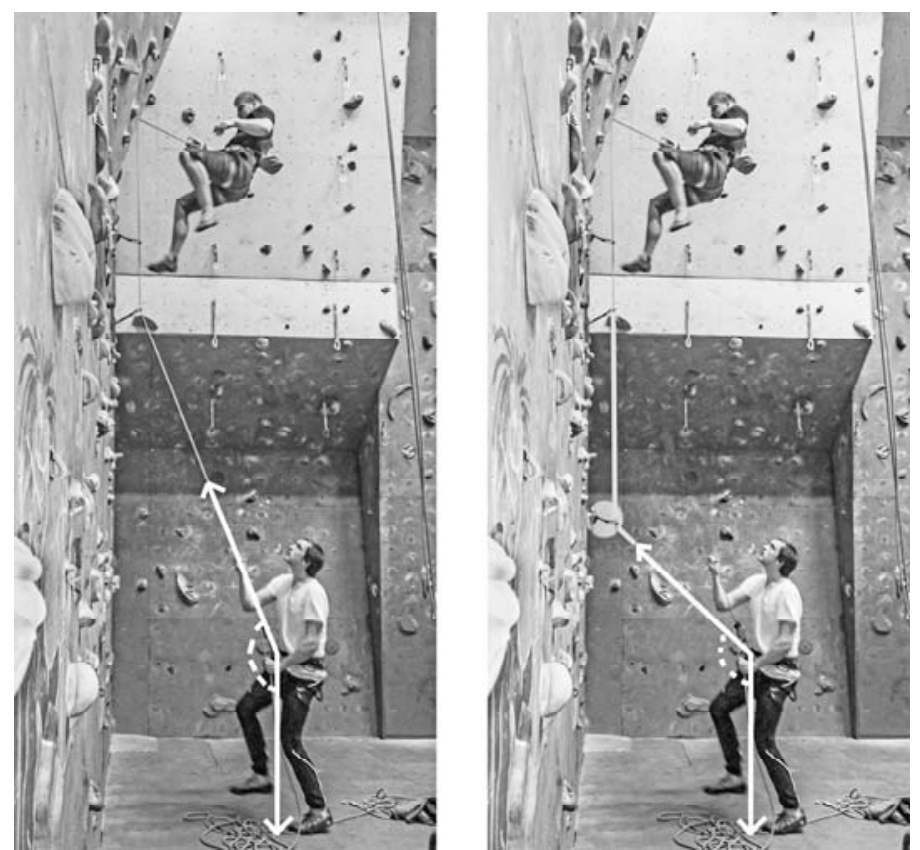

Figure 4. The first two steps in the falling situation in Lesson 2

The students also had to experience being a belayer standing a bit away from the wall, as in the leftmost picture in Figure 4. The climber falls, and then the belayer is tugged up and into the wall. Both students were aware of what would happen, and in this case the belayer can use one foot to parry the bump when (s)he meets the wall. The rope-pulling situation is based on the example in 
Figure 2. Three climbing ropes were attached to a loop; the other end of each rope was tied to a fishing weight that was hooked on to a student's climbing harness. The students used the weights to ensure that they pulled with equal force. The intention behind Lesson 1 was that the students would experience relations between the forces' magnitudes and directions. These experiences would then contribute to their re-invention of relations between angles and vectors, in in line with Georgios, Panayotis and Athanasios (2005) and Poynter and Tall (2005). The research focus was on investigating the students' development of ideas that might support their explanations of where the belayer should be located and why.

In Lesson 2, the falling-and-belaying situation from Figure 1 was replaced by a three-step falling-andbelaying situation. Figure 4 shows the two first steps. When the first runner is placed lower on the wall the belayer is pulled harder towards the wall. In step three, the first runner is placed at the belayer's chest height, and the belayer is pulled directly towards the wall. The students experienced being pulled harder and harder towards the wall, but they did not experience why this happens. These climbing and belaying experiences constituted the students' common basis for the discussions afterwards. The angle between the rope from the belayer and the force of gravity decreases systematically because the first runner is placed lower and lower. Each student experienced belaying in the three situations. Afterwards they explained what happened through drawings and words. The research focus was on the students' explanations of how a change in angle influences the vectors' magnitudes. Lesson 3 was a summary and reflection lesson that started with watching a similar falling situation in the video 'Vectors in climbing' (Fyhn, 2009). Then the situations were to be repeated if necessary.

The data material consists of a) the students' writings and drawings from the lessons, b) video recordings from Lessons 2 and 3 (there was no video recorder available the first day), and c) the researchers' handwritten notes from the meeting one year afterwards. To be open to changes in plans due to how the lessons worked out, the data also include d) the researcher's handwritten notes from the lessons, her plans for the lessons, and the summaries she made immediately afterwards.

\section{Analysis}

The analysis part identifies Bishop's (1988) six basic activities in the students' work. The analysis also identifies the role of angles and students' manipulation of mental objects. The point is not to reveal the students' learning outcome; it is rather to reveal aspects of vectors that were considered during the study.

The students used different terms and phrases when describing their previous knowledge of geometry. This indicates trustworthy data. Stian: «Full knowledge of Pythagoras, geometrical figures, know something about cosine, sine, tangent;» Lars: «Know the Pythagorean Theorem;» and Øystein: «I know how to use cosine, sine and tangent (mostly). I am able to use the Pythagorean sentence». In addition, Stian had read a book about mathematics in everyday problems, and Øystein experienced geometry as an easy theme. When it comes to vectors, Stian wrote, «vectors are a force shown by an arrow. The length of the arrow shows how strong the force is and the direction shows which way the force works». This is almost a verbatim imitation of what his elder climbing ideals say in the introduction to the teaching video 'Vectors in Climbing' (Fyhn, 2009). Lars wrote: «I know nothing about vectors, but believe that this is going to be fun and instructive and a good excuse for climbing;" while Øystein wrote: «The only thing I know about vector arithmetic is that, as opposed to a usual coordinate system, you may find a coordinate in a three-dimensional room by three axes.» 
Lesson 1

In the beginning, there was not much difference between the boys' outcomes from the falling-andbelaying situation in Figure 1. Their explanations focused on the length of the rope segment between the belayer and the lowest runner on the wall. The word 'angle' was not mentioned. Their intuitive ideas seemed to deal with the length of this rope segment. In order to challenge this intuitive idea, the researcher asked them to describe the angle between the rope and the vertical line (Field notes, February $16^{\text {th }}$, 2009). She introduced the word 'angle', which the students were familiar with but did not mention. The word was introduced through a question, which they were able to answer. This question is an example of guiding, in Freudenthal's (1991) terms. Some discussion followed, but there was no clear outcome of the discussion. Figure 5 presents Lars' drawing from the end of the lesson.

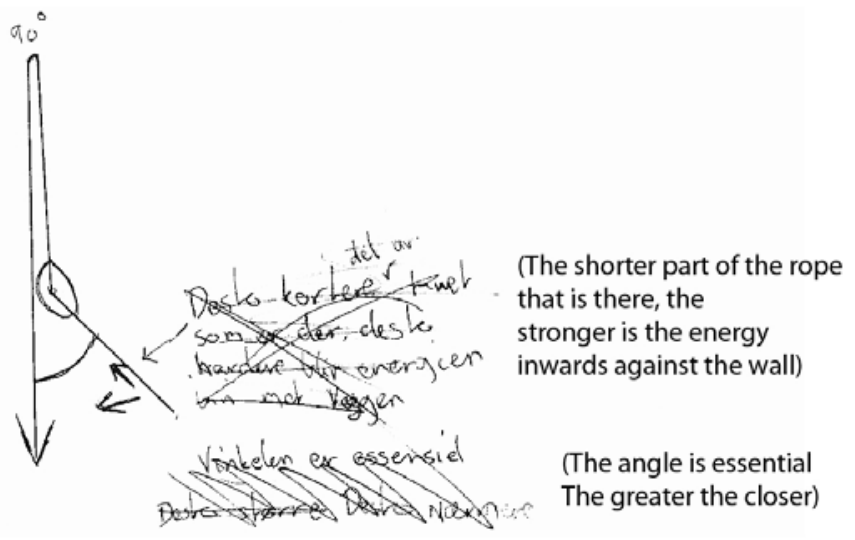

Figure 5. Lars' drawing from Lesson 1 (translated by the author)

Lars first described an idea involving the length of the rope between the belayer and the first runner. His second idea involved the importance of the angle between the rope from the belayer and the vertical rope in which the climber is hanging. The second idea was probably an outcome of the researchers' introduction of the word 'angle'. All his text was crossed out; perhaps he was not convinced of anything yet, or maybe he was unsure how to write down his ideas. His drawing shows a kind of intuitive decomposition of what he calls the 'energy' from the rope; he has marked two arrows that depart from the belayer. One arrow points towards the wall while the other points along the rope. This is manipulation of mental objects; Lars had constructed a mental object that he calls 'energy'. He carried out a mental manipulation of this object, which resulted in a decomposition; one part of the 'energy' works along the rope while the other one works towards the wall. The drawing shows that his manipulation of the mental object 'energy' involved some kind of relation between scalar and angle.

Location is prominent in Lars' drawing; the belayer and the first runner's locations are emphasised. Counting, on the other hand, does not play any role here. The drawing shows parts of a classic climbing situation in which a climber falls, which is an example of the activity playing; climbers climb for fun and not for any extrinsic reward. The climbers follow a set of agreed-upon rules, rules for the belayer and rules for the climber. Designing is what goes on when Lars makes the drawing; the drawing is a model of the situation. Explaining involves explaining what happens to the belayer, and the outcome of this activity is Lars' ideas of what happens and why. His first explaining concerns measuring; he is comparing the two magnitudes 'length of rope segment' and 'energy that works towards the wall'. Lars' text contains an idea about relations between these. His second explaining also concerns measuring; he is comparing the magnitudes of the angles. This explaining is highly relevant for climbers, because it is important that the belayer acts properly when a climber falls. Explaining like this does not consider what may be relevant to school mathematics. 
In the rope-pulling situation's first step, the students explained, «the angles between two of us are $120^{\circ}$, because 120 times three is $360 »$. This is an example of counting; when the three of them were organised regularly in equal distance from a centre, the ropes would shape the radiuses of three equal circular sectors. The students were aware that the circumference of a circle is $360^{\circ}$. The activity locating was important. Each student had to locate himself according to a given set of rules; the distance between each student had to be equal, and the scale on their fishing weights had to show the same magnitude. Following these rules is playing. The location of students according to the rules resulted in designing a shape. Afterwards they drew this shape on paper, and they were explaining in words what happened.

During the drawing session, the students came up with some questions regarding what they called 'the sideways forces' from Øystein and Stian in the rope-pulling activity: where did this force go? How could it just disappear? Øystein suggested that "the forces stabilised each other" (Field notes, February $\left.16^{\text {th }}, 2009\right)$. This is an example of manipulating the mental object force. His words can be interpreted as an attempt to decompose forces or as creating a mental object zero vector. Lars said that this reminded him of examples from a climbing book about how to place runners in outdoor climbing. This shows that he related the rope-pulling situation to his climbing experiences, even though the task in question was a closed task from a semi-reality. The students' intuitive ideas emerged as they were investigating the context of the problem. The researcher asked them how to mark the magnitude of a force without writing any numbers. Stian suggested "direction and length" (Field notes, February $16^{\text {th }}, 2009$ ); once again he cites his older climbing friends in the video verbatim.

While summing up what they had learned in Lesson 1, Lars wrote that "[w]hat we have learned is that the larger the angle is, the more energy will work on us" (Lars, field notes, February 17th, 2009). It is not clear whether he refers to the rope-pulling situation where the force from Stian and Øystein changed when the angle between their ropes changed. Maybe he referred to the falling situation, and tried to write that the angle between the rope and the vertical line influenced how hard he was pulled towards the wall. Øystein wrote that "[w] hat we have learned today is how angles and not lengths decide how the force is distributed" (Øystein, field notes, February $16^{\text {th }}, 2009$ ). Maybe he referred to the rope-pulling situation. It might be that he referred to the situation where the belayer was standing some distance away from the wall. When he was about to do the belaying he first objected: he knew from experience that this positioning was unwise. Stian had learned that "[i]t is the angle that decides how much you are being pulled inwards and upwards" (Stian, field notes, February 16 ${ }^{\text {th }}, 2009$ ). In the first part of the lesson, none of them was aware of the relevance of angles, but all of them wrote about it at the end of the lesson. All three of them seem to have learned something about the importance of angles when forces work in different directions.

\section{Lesson 2}

Stian explained the rope-pulling situation to Martine. When turning to the falling situation, the students just changed the plan: They wanted a top-rope fall to introduce the falling situation. A toprope fall is a fall where the climber is located below the last runner; usually the rope is fastened to the roof before the climber starts. One end of the rope goes from the roof and down to the belayer, while the other end goes down to the climber. There is less strain on the belayer in a top-rope fall than in a lead fall. The students' change of plan showed that they challenged the researcher's role; they changed the rules for the playing activity. They were designing a situation that could provide more information about what they were trying to find out. It is common climbing knowledge that in toprope falls the belayer will be lifted into the air only if the climber weighs discernibly more than the belayer does. The students compared lead falls and top-rope falls with regard to what happens to the belayer. This comparing is measuring. Locating plays an important role here because the last runner is located differently than in lead climbing. Explaining concerns why the belayer can stand still a bit away from the wall in top-rope falls but not in lead-climbing falls. The students organised the 
situation so that they had experiences that could confirm the difference between top-rope and leadclimb belaying.

Stian wrote about angles and vectors. Despite his use of formal mathematical terms, he did not mention anything about how angles and vectors are related: "The lesson focused more on angles and how diverse angles influence forces and vectors. I have started adding vectors" (Field notes, February $23^{\text {rd }}$, 2009). Øystein wrote: "I have studied how the attachment of the carabiner influences whether the belayer is being pulled towards the wall or not" (Field notes, February 23 ${ }^{\text {rd }}, 2009$ ), this is interpreted as referring to his behaviour as the first runner was placed lower and lower. He just described his exciting experiences, and wrote nothing about angles.

Martine had learned that "when the force from the rope works in opposition to the force of gravity, there is no movement between the climber and the belayer" (Martine, Field notes, February 23 ${ }^{\text {rd }}$, 2009). She refers to top roping. She made a statement based on her intuitive ideas of why the belayer is not lifted from the ground when the top-rope climber falls. She has created a mental object of a zero vector based on relations between direction and magnitude. Direction is an outcome of location, while magnitude is an outcome of measuring.

Regarding belaying in the lead-climb situation, she wrote, "The gravity works downwards. You fly less the closer to the wall you are standing, because of the angle between the ropes" (Field notes, February $23^{\text {rd }}$, 2009). Here she refers to the angle of the bent rope - the angle between the rope from the belayer and its continuation upwards after passing the first runner. Her sentence concerns locating; she describes a relationship between localisation of her body and the angle shaped by the rope.

Both Øystein and Martine wrote about locating of their bodies at the end of the lesson; they were explaining their bodies' more or less involuntary movements. Øystein experienced that the lower the first runner was located, the harder he was pulled towards the wall. Martine experienced that the angle of the bent rope influenced the extent to which she would fly towards the wall. They are explaining climbing experiences, not school mathematics. Stian's writing concerned mathematics and not climbing. He wrote that he would start adding vectors, but wrote nothing about how he planned to do it.

\section{Lesson 3}

The students watched a video presentation of 'The parallelogram method' (Fyhn, 2009), which is based on a situation similar to the falling situation. The force from the rope and the force of gravity were drawn and added in the video, presented in a picture similar to the last picture in Figure 1. The students objected quite immediately, claiming that they understood nothing at all. Their objection is interpreted as honesty, which in turn indicates trustworthy data. Then the students carried out, described, and explained the three steps of the rope-pulling situation once more. No new information was provided. This led to their formation of a hypothesis about relations between angles and the magnitudes of vectors. The students were guided to raise a mathematical problem that could lead them to explain why. Freudenthal $(1973$, p. 135) describes this as follows: «The problem should grow out of the situation, and the child should learn to recognize the problem in the situation. Raising a problem is mathematics, too».

By waving his hands above $B$ and $C$ in his figure, Stian explained step one: «...some of the force disappeared sideways» (Transcripts from video, March 9th, 2009). Pointing at B and C, Lars argued that «the sharper angle you have (points at B and C), the harder each of them has to pull, in order to make up for each other». These different ways of explaining the same thing are interpreted as honesty and trustworthy data. In addition, the boys had formed a mental object with the properties of a zero 
vector; the force 'disappeared sideways' and "to make up for each other". This mental object also has properties of vector decomposition. Lars's explanation concerns a relation between the angle between two vectors and their magnitudes. According to Georgios, Panayotis and Athanasios (2005), and to Poynter and Tall (2005), such relations are important.

Stian was locating his weaving hands symmetrically above the figure; the movement of one hand reflected the move of the other. He was conceptualising and symbolising the situation with a drawing, using words and his hands. Stian was designing different models of the situation; he used the symmetrical properties of his two hands to underline similar properties of the segments TC and TB in his drawing. Stian was explaining how forces disappeared, while Lars was explaining why the forces disappeared. The students did not pay any attention to the second step of the task; maybe they found it trivial since they had performed it before. The second step showed an example of a twodimensional zero vector. Because adding and subtracting along number lines are common activities in Norwegian school mathematics, the two-dimensional zero vector is expected to be trivial to the students.

The third step concerns the situation in which the angle between the ropes from $\mathrm{B}$ and $\mathrm{C}$ is $60^{\circ}$ and the ring still does not move. Two of them struggled with making mental calculations, and some mistakes caused friendly teasing and laughter in the group. Lars and Stian suggested halving TB and TC and bisecting $\angle \mathrm{BTP}$ and $\angle \mathrm{CTP}$. Their hypothesis concerns relations between angles and vectors, and their suggestion indicates an idea according to which there is a proportional relationship between a vector's angle and its magnitude. Martine falsified their hypothesis by saying, "... he he, OK ... then those two (pointing at figure) are pulling with $15 \mathrm{~kg}$ in total. And that equals what the person in the middle does. Isn't that a bit strange?" (Transcript from video, March 9nd, 2009). Lars immediately credited her, "well observed, well observed". After some more discussion Martine said, "... how much do they have to pull each (points at C) if they pull with $7.5 \mathrm{~kg}$ here (points at P)?" Then Stian replied, "... it has to be halfway between 7.5 and 15." The students kept their intuitive idea of halving while they were manipulating their idea of vector as a mental object. One interpretation is that the students possess some intuitive idea about a proportional relationship between angles and the magnitude of a force, and that this intuitive idea remains unchallenged.

The researcher asked if the students might draw a quadrilateral from some of the lines in the figure, and what such a figure would look like. Martine suggested a parallelogram, and they started drawing. The first drawing was no parallelogram, so they concluded that the figure was wrong and agreed to make a new one. Martine hesitated and asked, "What do we actually know?" Stian replied that TA and TP (in Figure 2b) have equal magnitudes.

The improved drawing was expected to verify the hypothesis, but it still did not look like a parallelogram. Instead of falsifying the hypothesis, Martine blamed the figure; "Since the measures and the angles are incorrect, the figure is not a parallelogram." Stian corrected her, "the measures are correct but the angles are not." Martine subsequently drew the rhombus in Figure 6. She pointed at the longest cathetus of a right-angled triangle, claiming that it was no longer than the hypotenuse "because that is how triangles are ..." She used previous mathematical knowledge as a tool for analysing the situation depicted in her drawing. Then she wrote the letter $x$ next to the shortest cathetus. Stian immediately asked what they were going to find out, but no one answered. The figure was an image of their idea, but they were not able to pose the problem yet. Lars asked what they had drawn, and the reply was "a more precise replication of the previous figure." 


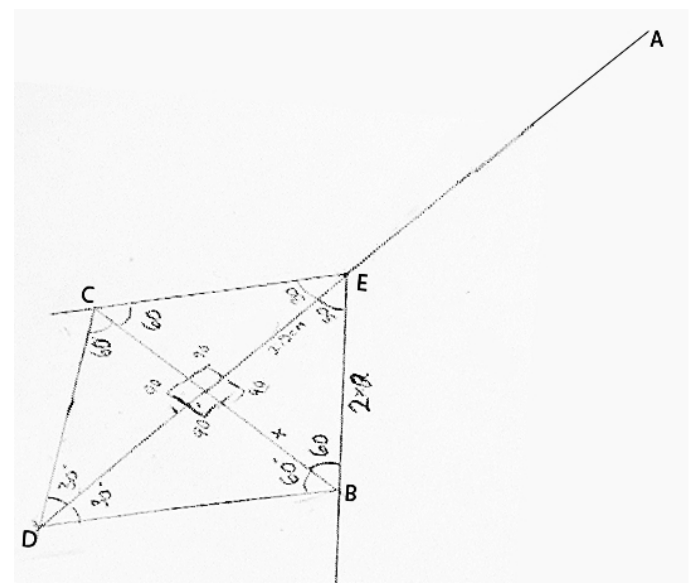

Figure 6. Martine's final rhombus

Martine asked, "How are we going to find it when we know only one of the sides?" Pointing at the longest cathetus, Lars then formed an equation, "We know that $2 x$, squared, minus $x$ squared, equals that one, squared." He also solved the equation and found that $\mathrm{EB}=\mathrm{EC} \approx 8,7$.

After the practical part of the rope-pulling exercise, the students returned to the video 'Vectors in Climbing' (Fyhn, 2009), where they were presented to the parallelogram method and the triangular method for adding vectors. The students watched and accepted. The researcher said that she expected this stuff to be difficult, and asked whether they were really sure that this was OK. It could be that the students were polite and did not want to disappoint the researcher, but this is unlikely since the students had objected spontaneously several times over the course of these three lessons. Most likely, they recognised some relationships between their discussions concerning the rope pulling and their experiences in and drawings of the falling situation.

\section{One year later}

The researcher was curious about what the students remembered afterwards. One year later, they were invited to meet in the climbing hall. Øystein was abroad that year, and Lars was busy with other things. Neither Martine nor Stian had encountered vectors in their lessons at school yet. The researcher asked what they remembered about vector addition. Their first answers came immediately. Stian referred to the rope-pulling situation, "We performed an experiment with a fishing weight and some ropes. I made a hypothesis that was wrong: if you chose the angle halfway between ... We calculated and found that the hypothesis was wrong, but I do not remember ..." Martine referred to the falling- and-belaying situation, "I remember that there was a force that changed when the angle changed. He was pulled inwards and upwards..." Stian added ..." you have more to counteract with when you are pulled directly upwards, because the weight of your own body counteracts in that case." Here he referred to top-rope falls as opposed to lead falls. Both of them referred to relations between angles and vectors in the falling situation. The conclusion is that Stian and Martine remembered what they had done one year ago and what went wrong. They referred to relations between vectors and angles, but not in detail. They did not mention the parallelogram law of vector addition.

\section{Discussion}

Dawson (2015) describes an instructional viewpoint concerned with getting students to learn how to learn rather than specific material. The aim is that students develop skills and attitudes for approaching problems in a rational way. During the VS, the students were asked to explain what happened. Their explanations were hypotheses. In Lesson 3, Martine falsified Stian's hypothesis, and the result was an immediate discussion that made him formulate a new hypothesis. In Lessons 1 and 
2, Stian's words are interpreted as showing that he tried to come up with what Dawson calls 'facts, results and definitions'. In Lesson 3, he was approaching the problem in a rational and critical way. When his conjecture was refuted, he came up with a new conjecture after a short discussion with the other students. The analysis of Lesson 3 indicates that the rope-pulling situation may support students' learning how to learn.

According to Dewey (1998/1933), the origin of thinking is some instance of perplexity, confusion or doubt. Given a difficulty, the next step is to form some tentative plan or project. In the VS, the climbers' challenge was to explain what happens when a climber falls and why the belayer has to stand close to the wall in lead climbing. Dewey calls this 'the first phase', where the thinking does not need to be reflective. If the climbers jump at a conclusion without weighing its premises, they may not engage in any inquiry. "One can think reflectively only when one is willing to endure suspense and to undergo the trouble of searching," (p. 16). Reflective thinking occurs when the examination turns into inquiry. To be genuinely thoughtful, the climbers need to undergo a state of doubt that stimulates thought inquiry. They need to find justifying reasons before they accept an idea. This is what the climbers did; they explained the reasons for their beliefs and then challenged each other's claims.

\section{Closing words}

The VS intends to give the students some situations that may support their understanding of a wellknown real-life climbing problem: where to locate yourself when belaying. The rope-pulling situation was added because the researcher expected that investigating a similar question in a different situation might support the students' understanding. In both cases, the question was how the force from a rope changes when a person is located in a different spot. There was a risk that this semi-reality context would have a negative effect, that the students would not grasp any similarities. In Lesson 3, the data showed that the symmetry properties of the illustration in Figure $2 b$ supported the students' understanding of the situation. When Stian explained the situation, he used three different representations to describe the same phenomenon; his hands, his words and his drawing. This indicates that the rope-pulling situation has a certain quality as teaching material; it is line with Sandoval and Possani (2016), who suggest that designing teaching material where students become aware of different representations, together with appropriate treatments and conversions, will promote students' flexibility and contribute to a richer understanding.

The VS is in line with Georgios, Panayotis and Athanasios (2005), who emphasise a focus on relations between angles and vectors in teaching. It is also in line with Poynter and Tall (2005), whose broader teaching goal is to create conceptual knowledge with a relational understanding of the concepts. In order to research what the use of climbing as an introduction to vectors reveals about students' development of a vector concept, Lesson 3 revealed an intuitive idea about a proportional relationship between a vector's angle and its magnitude. This idea needed to be challenged. An answer to this paper's research question is that the use of climbing as an introduction to vectors reveals the importance of students' development of two aspects of vectors before the parallelogram law of addition is introduced. These are a) relations between angles and vectors, and b) the zero vector.

\section{Acknowledgements}

The positive attitude and engagement shown by Lars, Øystein, Stian and Martine was important for the successful execution of this study and for providing me some interesting educational experiences. Per Øystein Haavold has provided some useful comments on the methods section. Morten Blomhøj has contributed with constructive questions related to several sections of the paper. 


\section{References}

Andersen, T., Jasper, P., Natvig, B. and Viken F. (2007). Giga. Matematikk R1. Programfag i studiespesialiserende utdanningsprogram. Oslo, Norway: N.W. Damm \& søn AS.

Bishop, A. J. (1988). Mathematics Education in its Cultural Context. Educational Studies in Mathematics, 19, 179-191.

Dawson, S. (2015). Learning mathematics does not (necessarily) mean constructing the right knowledge. For the learning of mathematics, 35 (3), 17-22.

Dewey, J. (1998). How we think. A restatement of the relation of reflective thinking to the educative process. Boston/New York: Houghton Mifflin Company. Original work published 1933.

Eriksen, T. Hylland (2001). Kultur, kommunikasjon og makt [Culture, communication and power]. In T. Hylland Eriksen (Ed.). Flerkulturell forståelse [Multicultural understanding]. Second edition (pp. 53-66). Oslo, Norway: Universitetsforlaget.

Freudenthal, H. (1973). Mathematics as an Educational Task. Dordrecht, Netherlands: D. Reidel.

Freudenthal, H. (1983). Didactical Phenomenology of Mathematical Structures. Dordrecht, Netherlands: D. Reidel.

Freudenthal, H. (1991). Revisiting Mathematics Education. China Lectures. Dordrecht, Netherlands: Kluwer Academic Publishers.

Fyhn, A. (2006). A climbing girl's reflections about angles, Journal of mathematical behaviour, 25, 91-102.

Fyhn, A. B. (2008). A climbing class' reinvention of angles, Educational studies in mathematics, 67 (1), 19-35.

Fyhn, A. B. (2009). Video: Vectors in climbing, NDLA Norsk Digital Læringsarena [Norwegian Digital Learning Arena]. http://ndla.no/nb/node/46170?from_fag=56, (accessed May 2016).

Fyhn, A. B. (2011). Introduksjon til vektorer i norske matematikklæreverk og i en klatrefilm. NOMAD Nordic Studies in Mathematics Education, 16 (3), 5-24.

Fyhn, A. B., Nutti, Y. Jannok, Eira, E. J. Sara., Børresen, T., Sandvik, S. O. and Hætta, O. E. (2015a). Ruvden as a basis for the teaching of mathematics: A Sámi mathematics teacher's experiences. In E. S. Huaman and B. Sriraman (Eds.), Indigenous Universalities and Peculiarities of Innovation. Advances in Innovation Education (pp. 169-186). Rotterdam/Boston/Taipei: Sense Publishers.

Fyhn, A. B., Dunfjeld, M., Dunfjeld Aagård, A., Eggen, P. \& Larsen, T. M. (2015b). Muligheter og utfordringer ved sørsamisk ornamentikk i matematikkfaget [Possibilities and challenges with including south Sámi ornamentation in school mathematics], Tangenten, 26 (4), 5-11.

Fyhn, A. B., Harbrecht, J. and Kristiansen, J. (under review a). Utforskende mathematikk på musikklinja [Investigative mathematics in the music program]. Under review by Tangenten - tidsskrift for matematikk i skolen. Bergen, Norway: Caspar.

Fyhn, A. B., Teig, V. T. and Pedersen, S. S. (under review b). Grunnleggende matematisering i småskolen [Basic mathematisation in lower grades]. Under review by Tangenten - tidsskrift for matematikk i skolen. Bergen, Norway: Caspar.

Gay, G. (2016). Cultural diversity and multicultural education. Curriculum Inquiry, 49 (1), 48-70.

Georgios, P., Panayotis, S. and Athanasios, G. (2005). The role of angle in understanding the vectors. Paper presentation at The $4^{\text {th }}$ Mediterranean Conference on Mathematics Education, January 28-30. Palermo, Italy: http://www.math.uoa.gr/me/faculty/spirou/THE\%20ROLE\%20OF\%20ANGLE\%20IN\%20THE\%20UNDERSTANDING \%20OF\%20VECTORS.pdf, (accessed June 2016)

Harel, G. and Trgalová, J. (1996). Higher Mathematics Education. In A. B. Bishop, M. A. Clements, C. Keitel, J. Kilpatrick and C. Laborde (Eds.) International handbook of mathematics education (pp. 675-700). Dordrecht, Netherlands: Kluwer Academic Publishers.

Hayfa, N. (2006). Impact of language on conceptualization of the vector. For the learning of mathematics, 26 (2), 36-40.

Van Hiele, P. M. (1986). Structure and Insight. A Theory of Mathematics Education. Orlando, Florida: Academic Press.

Klausen, A. M. (1992). Kultur, mønster og kaos. [Culture, pattern and chaos] Oslo, Norway: Ad Notam Gyldendal A/S.

Maracci, M. (2006). On students' conceptions in vector space theory. In J. Novotná, H. Moraová, M. Krátká and N. Stehlíková (Eds.) Proceedings 30 th Conference of the International Group for the Psychology of Mathematics Education, Vol 4, pp. 129-136.

Poynter, A. and Tall, D. (2005). What do mathematics and physics teachers think that students will find difficult? A challenge to accepted practices of teaching. Proceedings of the sixth British Congress of Mathematics Education, 128-135. http://www.bsrlm.org.uk/IPs/ip25-1/BSRLM-IP-25-1-17.pdf, (accessed June 2016).

Rosenbloom, P. C. (1969). Vectors and symmetry. Educational Studies in Mathematics, 2, 405-414.

Sandoval, I. and Possani, E. (2016). An analysis of different representations for vectors and planes in $\mathbb{R}^{3}$. Educational Studies in Mathematics, 92, 109-127.

Yin, R. K. (2009). Case Study Research. Design and Methods ( $4^{\text {th }}$ ed.). Thousand Oaks, California: Sage Publications Inc. 